\title{
TECNOLOGIAS EDUCACIONAIS DE ENSINO-APRENDIZAGEM NA FORMAÇÃO DO PROFISSIONAL ENFERMEIRO
}

\author{
Sandra de Nazaré Costa Monteiro, Manuela Costa Melo \\ Departamento de Enfermagem \\ Escola Superior de Ciências da Saúde - ESCS \\ Brasília - Distrito Federal, Brasil \\ E-mail: sncmonteiro@yahoo.com.br, melomanuela91@gmail.com
}

Resumo: A metodologia ativa apresenta desafios por possibilitar o rompimento com o ensino tradicional e o resgate da formação de profissionais competentes e comprometidos. Objetivou-se relatar a experiência de enfermeiros no curso de capacitação à prática Docente na graduação em Enfermagem, na Escola Superior em Ciências da Saúde, localizado em Brasília, Distrito Federal. Utilizou-se a demonstração das atividades ocorridas nas Unidades Educacionais da Dinâmica Tutorial, Habilidade Profissional de Enfermagem e o Portfólio de Formação, que constituem metodologias inovadoras e tecnológicas para a educação em enfermagem. A imersão no curso de capacitação proporcionou apropriação de novas tecnologias e incentivou a produção de conhecimento no processo de ensino-aprendizagem.

Palavras-chave: tecnologia, docente, metodologia ativa, tecnologias educacionais, ensinoaprendizagem.

\section{EDUCATIONAL TECHNOLOGIES IN TEACHING-LEARNING IN THE PROFESSIONAL NURSE TRAINING}

Abstract: The active methodology presents challenges for enabling a disruption with the traditional teaching and the rescue of training competent and committed professionals. The study aims to report the experience of nurses in the training course of teaching practice on nursing undergraduate, at the School of Health Sciences, located in Brasilia, Federal District. We used the statement of activities that occurred in the Educational Units of the Tutorial Dynamic, Professional Nursing Skill and the Training Portfolio, which together form innovative and technological methodologies to nursing education. The immersion in the training course provided appropriation of new technologies and encouraged the production of knowledge in the teaching-learning process.

Keywords: technology, teaching, active methodology, educational technologies, teachinglearning.

Recebido em 30/09/2015. Publicado em 30/03/2016. 


\section{INTRODUÇÃo}

O Sistema Único de Saúde (SUS) é identificado como ordenador da formação em saúde, e a instituição da Diretriz Curricular Nacional (DCN) no intuito de integrar a formação profissional ao SUS, evidenciou uma maior aproximação entre a formação em saúde, atenção e gestão, com o maior provedor da assistência à saúde no país, porém a contextualização de sua participação não se apresentava de forma clara na operacionalização dos rumos e das ações no ensino. Com esse intuito, em 2001, a DCN ocasionou movimentação nas Instituições de Ensino Superior (IES) em busca de mudanças nos cursos de graduação dos enfermeiros em todo país, na busca de cumprir as novas propostas de orientação e concepção curricular em seus Projetos Pedagógicos (PP). Fortalecendo assim, a necessidade de desenvolver uma formação do profissional de saúde relacionando o saber-conviver, saber-ser, saber fazer e saber-saber (NEVES \& BARROS, 2014).

Estudo realizado acerca da aderência de cursos de graduação em enfermagem às DCNs, na perspectiva do SUS, identificou que os discentes acreditavam que a articulação entre as instituições formadoras e o SUS acontece no decorrer das práticas e nos projetos de pesquisa e extensão, porém, ainda há a necessidade de maior fortalecimento do processo de formação do enfermeiro nos cenários de práticas na rede de serviços de saúde (FERNANDES et al, 2013). Nesse sentido é urgente à busca de uma metodologia que desenvolva um pensamento crítico reflexivo no estudante, nos quais os conhecimentos e competências vão se transformando velozmente, tornando-se essencial pensar em uma metodologia para uma prática de educação libertadora, na formação de um profissional ativo e apto a aprender a aprender (MITRE et al, 2008). Por isso a necessidade de uma metodologia com base em pedagogias ativas na educação.

Assim, a Escola Superior em Ciências da Saúde (ESCS), na graduação em enfermagem, busca atender a essa prerrogativa, e tem desenvolvido práticas de ensino aprendizagem que envolve a interdisciplinaridade, o aprender a aprender, o cenário de aprendizagem diversificado, a articulação de saberes de forma significativa ao estudante e assim, ser utilizados para assistência à saúde nas demandas do SUS (LUCHESE, VERA, PEREIRA, 2010). A carga horária do curso é de 4.788 horas em quatro anos, na modalidade seriada anual. 
Possui o alicerçado nas DCN e o seu PP formulado na proposta de perfil do egresso com visão generalista, humanista, crítico, reflexivo, ético e cidadão. A escola utiliza as Metodologias Ativas (MA) de ensino-aprendizagem, nas modalidades da Metodologia da Problematizacao (MP) e Metodologia da Aprendizagem Baseada em Problemas (ABP). Pertencente à própria Secretaria de Estado de Saúde do DF (SES-DF) busca uma conexão integrativa entre ensino e serviço visualizado pela inserção do estudante de forma precoce, ainda no primeiro semestre.

A MA apresenta inúmeros desafios e implicações, dos quais se sobressaem: a possibilidade de rompimento com o ensino tradicional e a necessidade de resgate da formação de profissionais competentes para recuperar importância do cuidado, que é a relação entre os sujeitos. E na mudança desse contexto educacional, faz-se necessário a utilização de novas tecnologias com abordagens pedagógicas progressivas com a utilização de ferramenta necessária ao desenvolvimento e consolidação das metodologias ativas, desenvolvidas a partir de uma sistematização criteriosa de etapas no processo ensino-aprendizagem. Nesse contexto a tecnologia no desenvolvimento profissional docente em enfermagem é emergente e vista como uma ferramenta necessária para o desenvolvimento e consolidação das MAs, desenvolvidas a partir de uma sistematização criteriosa de etapas no processo ensino-aprendizagem.

Estudo realizado usando um referencial que vem sendo incorporado na área da saúde classificam a MA como uma tecnologia leve-dura. As autoras desta proposta, ao analisarem as tecnologias em saúde, iniciam referindo que a palavra tecnologia é comumente relacionada às máquinas e que este entendimento tem dificultado bastante a nossa compreensão de que, quando falamos em trabalho em saúde, não estamos nos referindo apenas ao conjunto dos equipamentos ou tecnologias duras utilizados nas intervenções diagnósticas ou terapêuticas. Argumentam que é preciso olhar com atenção, pois o conjunto de intervenções assistenciais vai além das diversas máquinas utilizadas (MERHY \& ONOKO, 2007). As tecnologias podem ser classificadas em leve quando falamos de relações, acolhimento, gestão de serviços; em leve-dura quando nos referimos aos saberes bem estruturados, como o processo de enfermagem; e dura quando envolvem os equipamentos tecnológicos do tipo máquinas, as normas (SILVA \& LOURO, 2010).

No conjunto de equipamentos, instrumentos e saberes profissionais estão presentes a tecnologia leve-dura: leve por conter um saber que as pessoas adquiriram e que está inscrito na sua forma de 
pensar as situações de saúde e na sua maneira de organizar uma atuação sobre elas; e dura na medida em que é um saber-fazer bem-estruturado, bem-normalizado e bem-protocolado. Além destas duas situações tecnológicas abordadas, ainda trabalhamos com a tecnologia leve, ou seja, aquela que se produz através do trabalho vivo, entendido como processo das relações. Entende-se esta tecnologia como um encontro entre pessoas que atuam umas sobre e com as outras, criando espaços de intersubjetividade, onde acontecem os momentos das falas, escutas e interpretações, nos quais há a produção de uma acolhida ou não das intenções que estas pessoas colocam neste encontro (LOPES; SAUPE; MASSAROLI, 2008).

Diante do exposto, o presente estudo teve como objetivo relatar a reflexão das atividades vivenciadas por enfermeiros, na formação docente, conhecendo as formas de tecnologia em educação como ferramenta essencial de aprendizagem.

\section{METODOLOGIA}

Essa pesquisa consistiu em um relato de experiência que descreve aspectos vivenciados pelas autoras, na oportunidade de um curso de capacitação à prática docente para ESCS Enfermagem. Trata-se de um olhar qualitativo, que abordou a problemática desenhada a partir de métodos descritivos e observacionais. Para atuar como docente na ESCS/Enfermagem faz-se necessário que o enfermeiro realize um curso de $40 \mathrm{~h}$ de capacitação à prática docente. Esse curso de capacitação resultou nesta reflexão que aconteceu em outubro de 2014, nas Unidades Educacionais (UE) de práticas de enfermagem, da ESCS, situado na cidade de Brasília, Distrito Federal.

O curso ocorreu em três momentos, primeiro momento aulas teóricas em sala de aula, com informações sobre a dinâmica do Curso de Enfermagem e a utilização da MA; segundo momento, observar, na prática, a aplicação da pedagogia ativa; no último momento, o processo avaliativo por meio do Portfólio Formativo. Utilizou-se para estimular e desenvolver o ensino-aprendizado de docentes as seguintes informações: observação reflexiva e crítica, tomando por referência as aprendizagens adquiridas nas duas UE, a Dinâmica Tutorial (DT), que utilizada a ABP; e a Habilidade Profissional de Enfermagem (HPE), que utiliza MP. 
Os cenários práticos escolhidos foram: na observação da $A B P$, sala de aula da própria Escola; e na MP, cenário da saúde clinica da criança e do adolescente, o Hospital Regional da Asa Norte (HRAN). Ambas associam conteúdos da área do conhecimento das ciências biológicas, ciências humanas, sociais e de enfermagem, amparada na transdisciplinaridade e interdisciplinaridade, e com os eixos transversais à ética, ao processo saúde-doença, à comunicação e ao trabalho em equipe, que conjuntamente constituem metodologias inovadoras e tecnológicas para a educação em enfermagem.

\section{RESULTADOS E DISCUSSÃO}

Iniciamos o Curso de capacitação à prática Docente em Enfermagem da ESCS com nove encontros presenciais, cinco encontros em UE com atividades práticas e a elaboração do portfólio de formação. Acompanhamos os estudantes e os tutores desenvolvendo as práticas baseados no ABP e na MP. A formação de enfermeiros, na ESCS, possui como base no currículo por competências. Faz-se necessário que haja além de conhecimento teórico, habilidade e destreza prática na realização das mais diversas atividades inerentes à profissão, ou seja, aprender a ser - autonomia -, conhecer - assimilar conhecimentos-, fazer - adquirir conhecimentos e praticar-, conviver e trabalhar juntos - compreender a inter-relação.

O termo competência é a combinação de atributos, entre os quais destacam: domínio cognitivo e psicomotor, habilidades, atitudes e domínio afetivo. Com isso é possível adquirir visão ampla do campo de atuação. Desse modo, o agir competente, conforme as Normas da Resolução no 4/99, das DCNs, realiza-se pela "capacidade de mobilizar, articular e colocar em ação valores, conhecimentos e habilidades necessários para o desempenho eficiente e eficaz de atividades requeridas pela natureza do trabalho". Tanto na ABP quanto na MP, a utilização de problemas é o processo natural de aprendizagem significativa da vida real no processo de construção do conhecimento. Os problemas, ao se oferecer à análise como métodos permanentes solicitam um treinamento acerca da busca das informações relevantes e da capacidade de analisá-las, o que vai possibilitar maior fixação da aprendizagem, tanto para a formação acadêmica quanto o aprimoramento dos profissionais, dentro dos padrões educacionais esperados para o contexto e para a realidade social (GEMIGNANI, 2012). 


\subsection{Dinâmica Tutorial (DT)}

A Dinâmica Tutorial é uma atividade obrigatória realizada em grupo. Os grupos tutoriais são compostos por um tutor (docente) e oito a dez estudantes. Conforme a semana padrão da série, o grupo tutorial se reúne duas vezes por semana, para trabalhar os problemas de cada módulo. A cada problema são eleitos, entre os estudantes, um coordenador e um secretário. Cada estudante deve exercer pelo menos uma vez cada uma dessas funções, durante a realização do módulo.

É a base do método do ABP. Os estudantes são apresentados a um problema pré-elaborado pela Comissão de Elaboração de Problemas. Esse problema deverá atender a determinações curriculares e, dentro de um módulo temático, abordar um tema do conhecimento. Dessa discussão os alunos deverão formular objetivos de aprendizado. Um problema bem formulado encaminha o grupo de estudantes a eleger objetivos de aprendizado análogos aos imaginados pelos especialistas das várias disciplinas como necessários para o crescimento cognitivo do aluno dentro daquele tema específico. Dentre os estudantes um será o coordenador e outro o secretário daquela dinâmica, papéis que serão alternados entre os demais para propiciar que todos sejam coordenadores ou secretários. Os outros estudantes participantes do grupo deverão se esforçar para realizarem uma boa discussão do problema, de forma metódica, respeitando as diretrizes do coordenador do grupo.

Na ABP, o professor tem papel importante, que é permitir que o estudante aproprie-se do problema de forma independente e autônoma. Esta metodologia requer um esforço dos professores no sentido de propiciar modelos e cenários de ensino que permitam o trabalho e a aprendizagem em níveis adequados de complexidade e relevância (GEMIGNANI, 2012). Pensar numa pedagogia inovadora é pensar numa educação crítica, transformadora, cujos conhecimentos devem ser construídos de forma dinâmica, coletiva, cooperativa, contínua, interdisciplinar, democrática, participativa, transdisciplinar, pois só assim se pode contribuir para o processo de conscientização dos sujeitos para uma prática social emancipatória, uma educação libertadora, condição para a construção de sociedades sustentáveis (MILANI; PICANÇO; GEMIGNANI et al., 2009; GEMIGNANI, 2012).

Nossa atuação enquanto capacitandos objetivou experimentar como ocorre a MA na discussão de um módulo temático, por meio da DT e as contribuições que o tutor dentro da aprendizagem 
significativa pode oferecer para a elaboração e construção do conhecimento. A princípio, nossa atuação era apenas para observar como a metodologia se desenvolve no cenário prático, porém aos poucos fomos tornando parte do grupo.

Nesses termos dentro de cada módulo temático são estabelecidos objetivos de aprendizagem que refletem os objetivos gerais dos módulos, ondes os estudantes alcançam estes objetivos por meio da discussão dos problemas de cada unidade didática, durante as DT, desenvolvidas por meio da metodologia de ABP utilizam-se os seus Sete Passos: esclarecer termos e/ou expressões desconhecidas no problema; definir o problema a ser discutido; análise e troca de conhecimentos sobre o problema ("chuva de ideias"); revisão dos passos anteriores, com sistematização das hipóteses; definição dos objetivos de aprendizagem; levantamento de recursos de aprendizagem e estudo individual; discussão e resolução do problema a partir da revisão do passo anterior, à luz dos conhecimentos adquiridos (NEVES \& BARROS, 2014).

A estratégia educacional central do currículo ABP é a discussão de situações - problema ou casos clínicos em DT, que além de facilitar o processo de aquisição de conhecimentos, contribuem para o desenvolvimento de outros atributos na formação do estudante, entre eles: habilidades de comunicação, trabalho em equipe, solução de problemas, respeito aos colegas e desenvolvimento de postura crítica (TOLEDO JÚNIOR et al, 2008).

A primeira observação realizada na DT foi iniciada a atividade com apresentação dos presentes em sala de aula, compreendeu oito estudantes e um professor. O inicio deste tutorial foi em sanar as pendências da aula anterior foi sanado, e iniciado o assunto do dia. Na sequência foram repassadas as referências bibliográficas utilizadas, bem como banco de dados eletrônico por cada estudante. Composto o cenário da DT pelo estudante-coordenador e estudante-secretário, a discussão começou, e o recurso didático utilizado foi o painel de vidro para anotações.

O tutor estimulou a discussão e avaliou o grau de interesse, reforçando pontos importantes da temática. O desenrolar do assunto ocorreu com a contribuição dos estudantes que demonstram domínio no assunto, além de esquemas elaborados no caderno e artigos impressos no meio eletrônico. Em alguns momentos houve discordância de opiniões e questionamentos acerca do aprofundamento de um assunto diferente ao abordado na temática do dia. O tutor interviu e 
esclareceu que pela densidade do tema é pertinente, mas o assunto deverá ser construído numa aula específica, a ser planejada posteriormente.

O tutor estimulou a construção do conhecimento onde todos e contribuiu com a elaboração da síntese do assunto abordado. Quando houver saturação das informações, significou que o debate chegou ao fim e que o objetivo foi alcançado.

Ao final do dia, no processo avaliativo, todos os estudantes se posicionaram com relação ao êxito da DT, avaliando com o conceito satisfatório ou insatisfatório. Em seguida o tutor avaliou cada estudante e expôs sua opinião. Nós como observadores do processo, sinalizamos que a dinâmica foi efetiva, interessante. Esse procedimento culminou no fechamento de um problema respeitando os 7 passos da ABP. Nossa impressão a cerca do método foi muito boa, evidenciamos a que a metodologia ativa realmente empodera o estudante ao conhecimento, estimulando-o na busca de outros conhecimentos para a construção do saber coletivo. Por esse motivo há uma atmosfera de otimismo e busca entre eles.

A segunda observação realizada na DT, acompanhamos a abertura de um problema. Feita pelo estudante-coordenador da DT que por duas vezes fez a leitura do problema, no intuito de fixar o aprendizado, e assim levantar problemas e hipóteses - memória do conhecimento prévio do estudante -, o tutor relembrou que durante a elaboração de problemas, a discussão esclareceu pontos importantes acerca do assunto, os estudantes sentirem-se satisfeitos com as respostas, 0 assunto não foi considerado um problema, passando-se para discussão de outro ponto temático relevante. O tutor dispõe de um instrutivo pedagógico disponibilizado pela coordenação do curso que Ihe direcionou para sequencialmente orientar os estudantes.

Houve discussão entre o grupo na construção das hipóteses com necessidade de interferência do professor na elaboração e compreensão das mesmas. Esses procedimentos caracterizam a abertura de um problema.

O Processo de ensino-aprendizagem faz parte de um todo. Um complexo modelo interativo, centrado na construção do saber do estudante e na diversidade. O estudante, enquanto indivíduo é o responsável pela transformação, mediada pelo professor em uma relação dialógica 
problematizadora. A partir de perguntas, constrói uma hipótese, busca a teoria, faz a intervenção, volta à realidade e problematiza novamente (OLIVEIRA \& BATISTA, 2012).

Nossa impressão nessa DT em específico foi que o processo ensino-aprendizagem alcançou sucesso, onde as informações, o empenho, dedicação e o conhecimento fizeram-se presentes. 0 ato crítico-reflexivo se faz presente em todo momento da discussão o que estabeleceu uma dinâmica interessante de aprendizagem. Neste sentido, tanto tutores quanto os estudantes também conceituam a avaliação na sessão tutorial como reflexiva, possibilitando um pensamento sobre as ações ou atividades realizadas, como possibilidade de diálogo com os demais indivíduos envolvidos - tutores e pares - e como motivadora do processo educacional, sendo vista como um momento que possibilita o incentive ao estudo e desenvolvimento de habilidades (OLIVEIRA \& BATISTA, 2012).

A ênfase do tutorial encontra-se no processo "aprender a aprender" e não na transmissão de conteúdo, com o intuito de favorecer o desenvolvimento do pensamento crítico, da capacidade reflexiva. O estudante participa ativamente da construção do próprio conhecimento, e o professor possui a função de propiciar meios e ambientes facilitadores.

\subsection{Habilidades Profissionais em Enfermagem (HPE)}

O HPE é uma Unidade Educacional fundamental na formação das competências esperadas do graduando de enfermagem em ações individuais e coletivas, colocando em prática as habilidades e as atitudes, a partir da realidade e das necessidades concretamente identificadas no cenário de prática. É a UE no qual a MP está vinculada à realidade de saúde da população, envolvendo ações de promoção de saúde, prevenção, tratamento das doenças e recuperação da saúde, em equipe multidisciplinar, tendo como meta a transformação social do momento vivenciado (NEVES \& BARROS, 2014).

As atividades são realizadas em unidades de saúde nos atendimentos primários e secundários de saúde, nos laboratório da escola e na comunidade. Esta UE está fundamentada no trabalho em pequenos grupos, entre 5 a 8 estudantes, na diversificação de cenários, visando o desenvolvimento pessoal e a assimilação, de forma gradual e progressiva, de habilidades técnicas 
e humanas que permitam ao estudante adquirir as competências necessárias ao exercício profissional em diferentes contextos.

Nosso acompanhamento ocorreu com os estudantes da $2^{a}$ série, na Saúde Clínica da Criança e do Adolescente, e incluiu conteúdos teóricos e práticos, que compõem a assistência de enfermagem em nível individual e coletivo prestado à criança e ao adolescente internados. Estavam presentes para as atividades sete estudantes e um tutor. Foram observadas as seguintes atividades: planejamento e estratégias de implantação do cuidado integral ao paciente, realização de avaliação das ações; integração com os serviços e com a comunidade.

A atividade começou com o discurso do tutor acerca da dinâmica diária, informando sobre os pacientes que ficariam sob a responsabilidade de cada estudante e sobre realização atividade lúdica a ser desenvolvida pelo grupo. Nesta série, o estudante já desenvolve habilidades práticas de enfermagem, realizando anamnese e exame físico da saúde clínica da criança, aplicação do histórico de enfermagem, desenvolvimento das anotações e evoluções de cada paciente assistido, e as proposições de diagnósticos e intervenções de enfermagem.

Para otimizar as atividades práticas, a tutora organizou o grupo em dois subgrupos, sendo o primeiro empenhado na assistência clínica, e o outro na realização da atividade lúdica. No lúdico, os estudantes poderiam aplicar a criatividade no emprego de técnica que estimulassem o brincar para as crianças e os adolescentes, e que os pais/responsáveis das crianças/ adolescentes hospitalizados, compreendessem a importância do brincar no desenvolvimento infantil.

$\mathrm{O}$ tutor pouco interferiu nas condutas dos estudantes. Atuou como facilitador da aprendizagem quando percebia a falta direcionamento e sinalizava os pontos chaves da metodologia ativa. Os objetivos da primeira atividade, assistência clínica, encontra-se no estudante apontar as alterações clínicas identificadas no exame físico; realizar a avaliação do crescimento e desenvolvimento; realizar as orientações de promoção e prevenção pertinentes ao quadro clínico, e identificar as questões familiares, sociais e educacionais. A descrição da evolução de enfermagem, de cada paciente assistido, esteve relacionada na identificação das Necessidades Humanas Básicas da Teorista de Enfermagem, Wanda Horta, e seguiu a técnica do SOAP - que 
indica dados subjetivos, objetivos, avaliação e planejamento. Teve como padrões de desempenho esperados satisfatório e insatisfatório (ALMEIDA; CANTAL; COSTA JUNIOR, 2008).

Na avaliação diária, o tutor apontou os pontos a serem revistos e sanados em loco, ao mesmo tempo em que o estudante também se auto-avaliou e pontuou itens a serem melhorados. A avaliação de desempenho, feita pelo tutor é realizada por meio do check-list com os seguintes pontos: compromisso, responsabilidade, iniciativa, autossegurança, capacidade de assistência e pontos importantes da avaliação clínica do paciente, envolvendo crescimento e desenvolvimento, nutrição, questão familiar, social, educacional, diagnósticos de enfermagem e notificações.

Para reforçar as informações da prática nesta avaliação devem ser descritos os processos morfofuncionais envolvidos, os aspectos sociais, os cuidados demandados entre outros descritores dependendo do conteúdo trabalhado no período, os estudantes que não atenderam adequadamente os descritores, levam sua avaliação para casa e têm a oportunidade de refazê-la para uma apresentação escrita e oral, chamada de "sessão devolutiva", possibilitando-se aos estudantes, que ele reconheça o que não aprendeu e faça um investimento neste sentido (ALBUQUERQUE; SILVA; TANJI et al, 2010).

O grupo que desenvolveu atividades lúdicas escolheu reunir, na sala da Classe Hospitalar, todas as crianças que tivessem condições de participar e realizaram, com música e violão, brincadeiras que lembravam a infância, como passe o balão, stop, continue a frase e danças. Ao final, em avaliação, com a tutora, puderam esclarecer a metodologia escolhida: opção pela dinâmica, tipo de recreação, objetivos, público alvo, material e métodos, local, horário, resultados e pontos positivos.

É importante lembrar que nas MAs, o estudante é o ator principal do processo educacional, que é dinâmico e estimula a construção do conhecimento por meio de uma aprendizagem crítica e autônoma. Considera-se toda a experiência de "vida" trazida pelo aluno e entende-se que ele é mais propenso a aprender quando experimenta uma necessidade de conhecer algo para si mesmo e para sua prática (MAMEDE, 2001).

A MA é uma concepção educativa que estimula processos construtivos de ação-reflexão-ação, em que o estudante tem uma postura ativa em relação ao seu aprendizado numa situação prática de 
experiências, por meio de problemas que the sejam desafiantes e the permitam pesquisar e descobrir soluções, aplicáveis à realidade. No caso das atividades práticas, esses princípios são norteados pela MP, com base no Arco de Charles Maguerez - ou Método do Espiral - por meio de cinco etapas: observação da realidade; identificação dos pontos-chave do problema; teorização que consiste na fundamentação teórica do problema; elaboração da hipótese de solução em que ocorre a busca da resolução do problema de forma crítica e criativa; aplicação das hipóteses de solução à realidade, ou seja, consiste em problematizar a realidade por meio da observação, teorização e aplicação de ações para solucionar os problemas identificados (FREIRE, 2008).

Na MP, segundo Charles Maguerez, o facilitador deverá conduzir os estudantes a observar a realidade. Em seguida, discutir em pequenos grupos conhecimentos prévios sobre a situação, propor uma reflexão e uma análise que conduzam à identificação dos pontos-chave do problema, teorizar, gerar hipóteses sobre as causas para a resolução do problema e aplicá-las à realidade (GEMIGNANI, 2012).

Ao final de cada dia de atividade em HPE, estudantes e docente realizam a avaliação formativa, de acordo com as orientações contidas no manual da ESCS (NEVES \& BARROS, 2014). Nesse encontro a avaliação formativa foi vista como satisfatória pelos estudantes, tutor e capacitandos. Percebemos o quanto foi importante o papel do tutor de não fornecer aos estudantes fórmulas prontas, e sim fomentar, instigar a reflexão para resultar em uma ação.

No desenvolvimento da assistência clínica, foi possível observar a aplicação do Miniexercício de Avaliação Clínica (Mini-CEx), utilizado na avaliação da competências clínicas. Trata-se de uma opção complementar de avaliação, que consiste numa observação estruturada da prática com guia de verificação ou check-list, na qual se atribuem escores de avaliação global para diversos itens de atitudes e habilidades, seguida de feedback, com duração total de 20-30 minutos. Pode ser repetida várias vezes para o mesmo estudante, aumentando a validade do método (NORCINI et al, 2003).

A avaliação de competências clínicas constitui etapa essencial na formação do estudante e deve ser feita pela observação direta do desempenho em situação real. Ao final da consulta, o professor conversa com o estudante sobre suas falhas e acertos, configurando a avaliação formativa 
(MEGALE et al, 2008). De uma forma geral os estudantes demonstraram desempenho, interesse e competência na execução das atividades. Como capacitandas, nos sentimos gratificada pela oportunidade de conhecer a metodologia e poder utilizá-la futuramente.

A Unidade de HPE torna possível o estudante transcender a dimensão biológica no ensino da saúde, valorizando os aspectos psicossociais e culturais que determinam as relações interpessoais e profissionais.

\subsection{Portfólio de Formação}

Nessa etapa do curso foi elaborado e entregue o Portfólio Formativo reflexivo, que é considerado como estratégica de ensino e instrumento essencial para a finalização do curso de capacitação à prática docente. O Portfólio demonstra a performance do capacitando em determinado momento, tendo como base documentos coletados em cenários reais, selecionados e justificados quanto aos conhecimentos previstos, para elaborá-lo adequadamente (ALVARENGA \& ARAUJO, 2006).

"Um portfólio é uma coleção especial dos melhores trabalhos organizados pelos próprios alunos" (VILLAS BOAS, 2012). A autora ainda considera o portfólio como um dos saberes a serem incorporados pelos professores-alunos e pelos mediadores, pois articula teoria e a prática, servindo como procedimento avaliativo, mas, sobretudo, como eixo organizador do trabalho pedagógico, em virtude da importância que passa a ter durante todo o processo.

O portfólio é um caminho mapeado pelo desejo de formar em uma visão de educação integral, que vê o sujeito como um todo, o que envolve seus muitos aspectos além do cognitivo, o emocional, transpessoal, inter e intrapessoal. Ao propor, em sua elaboração, uma autorreflexão, traz ao estudante a possibilidade de interiorização, tão prejudicada em uma sociedade assoberbada pelo acúmulo de informações, imagens e ocupações que comprimem o cotidiano e não favorecem a autorreflexão e o conhecimento de si. Na escolha dos trabalhos há uma auto-avaliação onde o sujeito se vê, pensa em si de modo crítico e cuidadoso, compreendendo seu contexto e funcionamento. Essa compreensão orienta novas ações, abre outros caminhos (OLIVEIRA \& ELLIOT, 2012).

A problematização implicou na interpretação e na percepção crítica da realidade, o que contribuiu no fortalecimento da identidade do sujeito-cidadão crítico (BORDENAVE, PEREIRA, 2004). Sendo 
assim, permitiu que os estudantes desenvolvessem um atendimento que valorizasse não apenas o biológico, como também o cuidado humanizado (PRADO et al, 2012; LIMBERG, 2013). Nesse sentido, as atividades desenvolvidas apontaram novas descobertas, tornando-se espaço oportuno para a construção coletiva de um saber na busca da formação de profissionais com habilidades e competências aliada ao senso crítico e transformador. A junção das três formas metodológicas utilizadas no curso, a ABP, a MP e a construção do portfólio formativo, culminou em estratégias tecnológicas leve-duras empregadas nas áreas da saúde e educação.

\section{CONCLUSÃO}

A imersão no curso de capacitação à prática docente nos proporcionou a apropriação de novas tecnologias e incentivou a busca de produção de conhecimento, associando recursos tecnológicos e estratégicos ao processo de ensino-aprendizagem na área da saúde.

Os estudos individuais, trocas de experiências, pesquisas na internet, compartilhamento de ideias e uma busca constante para compreender o funcionamento desse novo modelo de aprendizagem, surgiram muitas reflexões e questionamentos que oscilaram do conhecimento prévio a um entendimento mais elaborado por meio do exercício de construção da metodologia ativa.

Cada encontro em sala de aula ou em cenário de prática ocorreu de forma crítica-construtiva, e sem perceber fomos familiarizando com os termos ABP, MP a partir do Arco de Maguerez, dinâmica tutorial, habilidades profissionais de enfermagem e muitos outros termos novos e conflitantes quanto comparados ao método tradicional.

O curso atingiu seu objetivo e a partir do momento que percebemos uma aprendizagem significativa nas discussões e atitudes de cada participante. O aprender a ser, conhecer, fazer, conviver e trabalhar juntos é um exercício constante quando se trabalha com educação centrada em competências.

\section{REFERÊNCIAS}

ALBUQUERQUE, V.S.; SILVA, C.M.S.L.M.D.; TANJI, S. et al. Os espaços de construção do conhecimento e a avaliação no currículo integrado do curso de enfermagem do unifeso. R. pesq.: cuid. fundam. online. v.2, n.3, p.997-1008, 2010.

ALMEIDA, F.F,; CANTAL, C.; COSTA JUNIOR, A.L. Psicologia ciência e profissão. v 28, n.2, p.430-442, 2008. 
ALVARENGA, G. M.; ARAUJO, Z. R. Portfólio: conceitos básicos e indicação para sua utilização. Estudos em Avaliação Educacional, v. 17, n. 33, jan./abr. 2006.

BORDENAVE, J.D.; PEREIRA, A.M.P. Estratégias de ensino-aprendizagem. 25a ed. Rio de Janeiro: Vozes; 2004.

FERNANDES, J. D.; SILVA, R. M. O.; TEIXEIRA, G. A.; FLORENCIO, R. M. S.; SILVA, L. S.; REBOUÇAS, L. C. C. Aderência de cursos de graduação em enfermagem às Diretrizes curriculares nacionais na perspectiva do Sistema único de saúde. Esc Anna Nery, v.17, n. 1, p.82-89, 2013.

FREIE, P. Pedagogia da autonomia: saberes necessários à prática educativa. São Paulo: Paz e Terra, 2008.

GEMIGNANI, E. Y. M. Y. Formação de professores e metodologias ativas de ensinoaprendizagem: ensinar para a compreensão. Fronteiras da Educação [online], Recife, v. 1, n.2, 2012.

LIMBERGER, J.B. Metodologias ativas de ensino-aprendizagem para educação farmacêutica: um relato de experiência. Interface (Botucatu) [online]. vol.17, n 47, p. 969-975. 2013.

LOPES, W.O.; SAUPE, R.; MASSAROLI, A. Visita domiciliar: tecnologia para o cuidado, o ensino e a pesquisa.Cienc Cuid Saude., v. 7, n.2, p.241-247, Abr/Jun. 2008.

LUCCHESE. R.; VERA, I.; PEREIRA, W.R. As políticas públicas de saúde - SUS - como referência para o processo ensinoaprendizagem do enfermeiro. Rev. Eletr. Enf. [Internet]. v.12, n.3, p.562-6. 2010.

MAMEDE, S. Aprendizagem baseada em problemas: características, processos e racionalidade. Fortaleza: Hucitec, 2001.

MEGALE, L et al. Avaliação de Competência Clínica pelo Miniex. Revista Brasileira de Educação Médica. v.33, n.2, p.166 -175, 2009.

MERHY, E.E.; ONOKO, R. Agir em saúde: um desafio para o público. São Paulo: Hucitec; 2007.

MILANI, A.H.; PICANÇO, A.C. JR; SOARES, E; GEMIGNANI, E.Y.M.Y. et al. Como promover a construção coletiva e o desenvolvimento do currículo a partir de uma visão sistêmica? In: CAMPOS, D.A. (org.) Docência no Cenário do Ensino para a Compreensão. São Paulo: UNICID, 2009.

MITRE, S. M.; SIQUEIRA-BATISTA, R.; MENDONÇA, J. M. G. et al. Metodologias ativas de ensino-aprendizagem na formação profissional em saúde: debates atuais. Ciência \& Saúde Coletiva, v.13, n. 2, p. 2133-2144, 2008.

NEVES, R.S.; BARROS, A.F. Manual de avaliação do curso de graduação em enfermagem / Brasília: Fundação de Ensino e Pesquisa em Ciências da Saúde / Escola Superior de Ciências da Saúde, P. 41, 2014.

NORCINI, J. J. et al. The mini-CEX: a method for assessing clinical skills. Ann Intern Med., v. 138, n. 6, p. 476-81, 2003.

OLIVEIRA, D. L.; ELLIOT, L. G. O Portfólio como Instrumento de Avaliação da Aprendizagem em Escola Montessoriana. Meta: Avaliação. Rio de Janeiro, v. 4, n. 10, p. 28-55, jan./abr. 2012

OLIVEIRA, V. T. D.; BATISTA, N. A. Avaliação formativa em sessão tutorial: concepções e dificuldades. Rev. bras. educ. med., Rio de Janeiro, v. 36, n. 3, 2012.

PIGATTO, L. M. M. A recreação como metodologia no processo de ensino Aprendizagem. RS. unifra.br, 2007.

PRADO, M.L; VELHO, M.B.; ESPÍNDOLA, D.S.; SOBRINHO, S.H.; BACKES, V.M.S. Metodologia Ativa na formação de profissionais de saúde. Esc Anna Nery, v. 16,n. 1, p. 172-77. 2012.

SILVA, R. C. L. ; LOURO, T. Q. A incorporação das tecnologias duras no cuidado de enfermagem em terapia intensiva e o desenvolvimento do discurso da humanização. Rev. enferm. UFPE on line, v.4, n.3, p.1557-1564. 2010.

TOLEDO JÚNIOR, A. C. C. et al. Aprendizagem baseada em problemas: uma nova referência para a construção do currículo médico. Revista Médica de Minas Gerais, Belo Horizonte, v. 18, n. 3, 2008.

VILLAS BOAS, B.M.F. Portfólio, avaliação e trabalho Pedagógico. $8^{\mathrm{a}}$ ed. Campinas, SP. Papirus, 2012. 